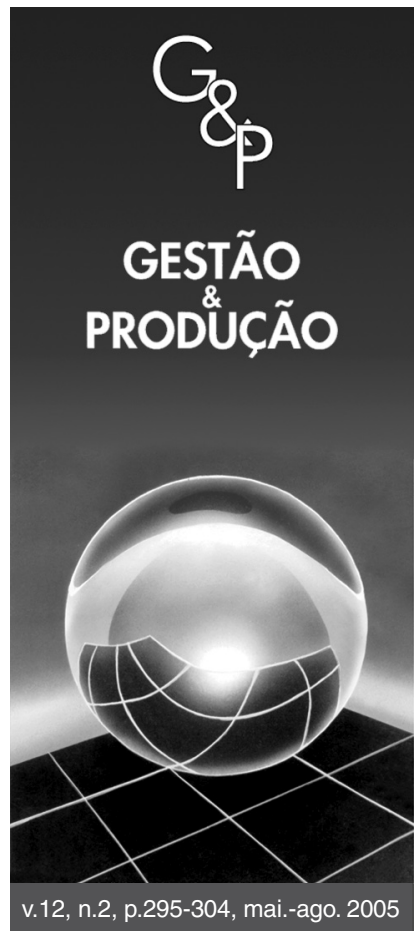

\title{
UMA CONTRIBUIÇÃO AO PROBLEMA DE COMPOSIÇÃO DE CARTEIRAS DE MÍNIMO VALOR EM RISCO
}

\author{
Celma de Oliveira Ribeiro \\ Leonardo Augusto Soares Ferreira
}

Departamento de Engenharia de Produção, Escola Politécnica da USP, Av. Prof. Almeida Prado, 128, Tr. 2 biênio, $2^{\circ}$ andar, CEP 05508-900, São Paulo, SP, e-mails: celma@usp.br, leonardo.ferreira@br.abnamro.com

\section{Resumo}

O trabalho propõe um modelo baseado em aproximação estocástica para composição de carteiras de ativos financeiros de mínimo risco. A medida de risco estudada, o Valor em Risco, é bastante utilizada na prática de gestão financeira como um sinalizador para tomada de decisão, porém poucas vezes é empregada para definir a composição ótima de carteiras em decorrência das dificuldades de implementação computacional. O modelo proposto permite que o problema de composição de carteira de mínimo Valor em Risco seja resolvido de uma maneira simples. O artigo analisa o desempenho do modelo em um problema de gestão de carteiras de ações no mercado brasileiro.

Palavras-chave: gestão de carteiras, finanças, otimização.

\section{Introdução}

O problema de mensuração e controle de risco financeiro tem motivado pesquisadores e tomadores de decisão a buscar indicadores capazes de identificarem quão arriscada é uma decisão. Uma fértil área de pesquisa desenvolveu-se na última década, tratando do problema de encontrar medidas de risco capazes de quantificar apropriadamente o risco de uma determinada posição assumida por um investidor. Na maioria das vezes, as medidas de risco presumem a existência de uma distribuição de probabilidade para os fatores de risco e avaliam momentos ou percentis da distribuição da função de perda.

A rápida evolução da área modelagem em finanças, aliada a um expressivo crescimento na disponibilidade de produtos financeiros no mercado, possibilitou o desenvolvimento de modelos matemáticos com relativa complexidade, visando responder a importante questão de como construir uma carteira de ativos de mínimo risco. Em essência, pretende-se minimizar uma medida de risco, para carteiras sujeitas a um conjunto de restrições, o que recai em problemas de otimização. A complexidade destes problemas depende fundamentalmente da medida de risco utilizada, da estrutura do conjunto de restrições e da natureza dos ativos envolvidos. Ativos financeiros como opções, por exemplo, introduzem dificuldades bastante grandes nos problemas, sendo necessário empregar modelos baseados em cálculo estocástico. Modelos dinâmicos, que analisam um horizonte temporal longo, tais como problemas de gestão de ativo-passivo (asset liability management) também levam a situações complexas. Mesmo no caso estático, ainda que se trabalhe com ativos de simples tratamento, há ainda que se considerar as dificuldades resultantes do uso de uma específica medida de risco.

Dentro deste escopo, a proposta do trabalho é analisar carteiras de ativos financeiros sujeitos a um único fator de risco, o risco de mercado, visando determinar carteiras de mínimo risco. Trabalhar-se-á dentro de um ambiente estático, de um único período, e serão tratados como medida de risco os percentis da distribuição de probabilidade das perdas da carteira. $\mathrm{O}$ foco do trabalho é o emprego 
de técnicas de aproximação estocástica para otimização de carteiras, utilizando a metodologia de superfície de resposta (Response Surface Methodology). Serão apresentados resultados computacionais obtidos a partir do uso desta técnica para minimização do risco de carteiras de ativos financeiros, tomando-se para tanto uma medida de risco não convexa.

\section{Percentis como medida de risco}

Historicamente, antes do desenvolvimento do conhecido modelo de Markowitz (1952), o risco financeiro era considerado um fator de correção dos retornos esperados e os retornos ajustados ao risco eram definidos $a d$ hoc. A principal inovação proposta por Markowitz foi a interpretação do retorno de uma carteira como uma variável aleatória cujo risco é avaliado por meio de sua variância, isto é, do segundo momento da distribuição. A partir desta abordagem, foi possível compreender que a diversificação reduz o risco, na medida em que carteiras que contêm ativos negativamente correlacionados possuem variância (risco) inferior à soma das variâncias (riscos) individuais. Um problema nesta abordagem é que a variância, ou desvio padrão, do retorno de uma carteira apresenta propriedades indesejáveis como a inadequação para avaliar situações de perdas extremas, situadas nas caudas da distribuição de probabilidade. Além disso, para que possa tratar a variância de uma maneira simples, pela covariância, o modelo pressupõe que a distribuição de probabilidade dos retornos é elíptica o que na prática não ocorre. Seguiram-se ao modelo de Markowitz, numa mesma linha, modelos que empregam medidas de risco definidas por outros momentos de distribuições de probabilidade, como o caso de Konno (Konno e Yamazaki, 1991), que propõe o uso do primeiro momento absoluto da distribuição como métrica.

A prática da gestão de risco mostrou que considerar o primeiro e segundo momentos da distribuição de probabilidade do retorno de uma carteira como medida de risco era insatisfatório, na medida em que não permite identificar anomalias ou mesmo a extensão de possíveis perdas com uma dada carteira. Motivado por diversos desastres financeiros envolvendo derivativos, nos quais as medidas tradicionais mostraram-se inadequadas, o Valor em Risco (VaR) surgiu, então, como medida de risco. Formalmente, o VaR pode ser definido como sendo a pior perda esperada ao longo de determinado intervalo de tempo, sob condições normais de mercado e dentro de determinado nível de confiança, ou seja, o VaR é um percentil da distribuição de probabilidade das perdas. A função de perda, no caso estudado, será dada pelo oposto do retorno da carteira.

A seguinte definição é apresentada por Acerbi e Tasche (2002). Sendo P uma medida de probabilidade asso- ciada a uma variável aleatória $\mathrm{R}$ que determina o retorno de uma carteira, o Valor em Risco, ao nível $\alpha$, é definido como:

$$
\begin{aligned}
& \operatorname{VaR}_{\alpha}=-\mathrm{q}^{\alpha}(\mathrm{R}), \\
& \text { em que } \\
& q_{\alpha}(R)=\inf \{w \in \Re \mid P(R \leq w)>\alpha\}
\end{aligned}
$$

Conceitualmente, a obtenção do VaR é simples, uma vez que é um percentil, porém, na prática, sua determinação depende de técnicas estatísticas de estimação o que introduz incertezas em seu cálculo. Há diversos métodos para estimação do VaR. O método de estimação paramétrico parte da premissa que a distribuição de probabilidade do retorno da carteira é normal, bem determinado a partir de sua média e variância, o que possibilita obter uma expressão analítica simples (Jorion, 1997). Também é possível utilizar técnicas computacionalmente intensivas, baseadas em séries históricas como bootstrapping ou mesmo simulação de Monte Carlo, nos quais se pressupõe que o histórico passado dos retornos reflete, de maneira adequada, o que deverá ocorrer no futuro. Outras técnicas fundamentadas na teoria de valores extremos ou em modelos de volatilidade estocástica também têm sido empregadas (Embrechts, 2000; McNeil, 1998; Szegö, 2002). Uma maneira de estimar percentis, denominada método da série histórica, consiste no uso de estatísticas de ordem. Sendo $R=\left\{r_{1} \ldots r_{T}\right\}$ um conjunto de observações do retorno de uma carteira, um estimador de percentil é dado por $M_{[\alpha T: T]}(R)$, em que $M_{[I: T]}$ é o mínimo de $R=\left\{r_{1} \ldots\right.$ $\left.r_{T}\right\}, M_{[T: T]}$ o máximo, e $M_{[\alpha T: T]}$ o $\alpha T$-ésimo valor em ordem crescente. Ou seja, segundo o modelo de série histórica, para o cálculo do VaR é preciso, inicialmente, selecionar uma amostra de retornos para a carteira, ordenar este valores e escolher um valor de acordo com a função $M_{[\alpha T: N]}(R)$, em que $\alpha$ é o nível de confiança desejado.

Há diversas considerações sob o ponto de vista prático que devem ser observadas pelo tomador de decisão, na escolha do método a ser empregado para estimar o VaR. A opção por uma particular metodologia de cálculo deve retratar uma necessidade da instituição (expressa em termos de nível de confiança) e também ser apropriada à particular carteira e tipos de ativos que a constituem. Ao estudar o VaR, é necessário, também, determinar o período para o qual deverá ser verificada a pior perda. Este período, denominado horizonte de tempo, está diretamente relacionado com o tipo de empresa e características da carteira em que o VaR será calculado. Em empresas em que os ativos financeiros são pouco negociados, utilizase um horizonte de tempo maior, como por exemplo, um mês. Já em instituições que possuem um alto giro dos ativos financeiros, é recomendável utilizar um horizonte de tempo menor, como um dia. Definido o horizonte de tempo é necessário estabelecer o nível de confiança, que indica a probabilidade de se verificar uma perda superior 
à indicada pelo VaR no horizonte de tempo considerado. A escolha deste valor está mais ligada às características de aversão ao risco das empresas: as mais conservadoras tendem a adotar níveis de confiança mais altos.

Na presença de instabilidades no mercado e de desvios significativos nas condições de normalidade, sabe-se que o VaR pode conduzir a ineficiências na gestão de carteiras. Além disso, esta medida não possui propriedades importantes como a subaditividade, não podendo ser classificada como uma medida coerente de risco (Artzner, 1999). Apesar destas limitações, esta medida se tornou um padrão mundial de avaliação de risco. Seu estudo justifica-se não apenas pela sua aplicabilidade, mas também em decorrência da complexidade do problema resultante, quando o VaR é utilizado na composição de carteiras.

\section{Composição de carteiras}

O problema de composição de carteiras está intrinsecamente relacionado aos conceitos de risco e retorno. Quando um operador do mercado financeiro ou administrador de empresas compõe uma carteira de ativos, o objetivo é basicamente obter o máximo de retorno possível, dado um nível aceitável de risco, ou obter o mínimo risco, fixado um nível de retorno. A preocupação das instituições com relação à composição de suas carteiras não é recente. O trabalho de Markowitz, publicado em 1952, tratou da questão de composição de carteiras de mínimo risco sob a ótica de modelos de otimização, e tornou-se um marco na histórica de finanças.

A literatura a respeito deste problema é extensa, ainda que recente. Há diversos trabalhos científicos que tratam do problema de determinação de carteiras de mínimo risco (Judice et al., 2003; Ribeiro et al., 2002; Pflug e Gaivoronski, 2005; Bertsimas et al., 2004) sob a ótica de otimização. Em particular, o problema de determinação de algoritmos eficientes para o problema de minimização de percentis, como é o caso do Valor em Risco, tem desafiado pesquisadores. Algumas técnicas têm sido propostas para analisar este problema por autores consagrados. Rockafellar e Uryasev (2000) apresentaram uma elegante formulação analítica para $\operatorname{VaR}_{\alpha}(\mathrm{x})$, o Valor em Risco escrito a partir de uma particular carteira $x \in \mathfrak{R}^{n}$, em um nível de confiança $\alpha$, que pressupõe o conhecimento a priori, da distribuição de probabilidade dos retornos. Pflug e Gaivoronski (2005) propôs uma aproximação polinomial para $\operatorname{VaR}_{\alpha}(\mathrm{x})$, pressupondo que os valores estimados são adequados para a modelagem, sem considerar a incerteza nas estimativas. Kibzun e Kipp (2001) analisam aproximações discretas do problema.

Em linhas gerais, pretende-se estudar o problema de composição de carteiras de mínimo VaR por meio de um modelo tratável computacionalmente, mesmo em situações nas quais não se presume normalidade. Formalmen- te, o problema de interesse consiste em determinar uma carteira $x \in \mathfrak{R}^{n}$ que forneça o menor Valor em Risco, ou seja, encontre, se existir, $x^{*} \epsilon \arg \min \operatorname{VaR}_{\alpha}(x)$,

$$
X\left(R_{o}\right)=\left\{x \in \Re^{n} \mid \sum_{j=1}^{n} x_{j}=1 \quad \sum_{j=1}^{n} E\left(r_{j}\right) x_{j} \geq R_{o} \quad x_{j} \geq 0 \forall j\right\}(2)
$$

em que $x \in \mathfrak{R}^{n}$ é o vetor que determina a composição da carteira, de forma que $x_{i}$ é o percentual do i-ésimo ativo presente na carteira. A constante $\mathrm{R}_{0}$ é o retorno mínimo exigido pelo investidor, e $\mathrm{E}\left(\mathrm{r}_{\mathrm{j}}\right)$ é o valor esperado do retorno do j-ésimo ativo.

Neste modelo as restrições são lineares e não causam problemas do ponto de vista de otimização, porém a função objetivo apresenta características que dificultam a implementação do modelo. Modelos que consideram as estimativas paramétricas do VaR como função objetivo são equivalentes ao modelo tradicional de composição de carteiras de Markowitz e podem ser facilmente resolvidos por técnicas de otimização quadrática (Luenberger, 1998), (Steinback, 2001). No entanto, a menos do caso em que o VaR é estimado a partir do pressuposto de normalidade da distribuição de probabilidade dos retornos, não há uma expressão analítica trivial que possibilite escrever $\operatorname{VaR}_{\alpha}(\mathrm{x})$. Ao utilizar estimadores para aproximação da função objetivo, o problema passa a ter diversos mínimos locais o que dificulta, e até mesmo inviabiliza, a otimização segundo os métodos de otimização tradicionais.

Apresenta-se a seguir um exemplo hipotético que ilustra algumas das dificuldades inerentes ao problema. Foram selecionadas duas composições de carteiras $x^{1}$ e $x^{2}$, a partir de 5 ativos, e foi gerada uma família de carteiras: $x(\lambda)=\lambda x^{1}+(1-\lambda) x^{2}, \lambda \in[0,1]$. Calculou-se o VaR para esta família de carteiras, utilizando-se o método de série histórica para um horizonte de um dia, a um nível de confiança de 95\%. Na Figura 1, estão representados os resultados, sendo que o eixo horizontal representa o valor de $\lambda$ e o eixo vertical o VaR.

Observa-se neste exemplo que, embora o ponto de mínimo global da função esteja próximo de 0.20 , há vários mínimos locais (alguns indicados por círculos). Além disso, pode-se observar, como no ponto $\lambda$, por exemplo, que a função possivelmente não é diferenciável e verificou-se empiricamente que as irregularidades aumentam com o número de ativos da carteira.

Para permitir a otimização desta função, pode ser utilizada uma técnica de aproximação estocástica, apresentada na seção que segue, que interpola um conjunto de observações. No exemplo apresentado na Figura 2, são utilizados 6 pontos para interpolação $\Lambda=\left\{\lambda_{1} \ldots \lambda_{6}\right\}$ e verifica-se que a aproximação resultante possui um comportamento bastante apropriado ao uso de algoritmos de otimização.

O emprego de técnicas de suavização em problemas de otimização com o intuito de reduzir o número de mínimos 


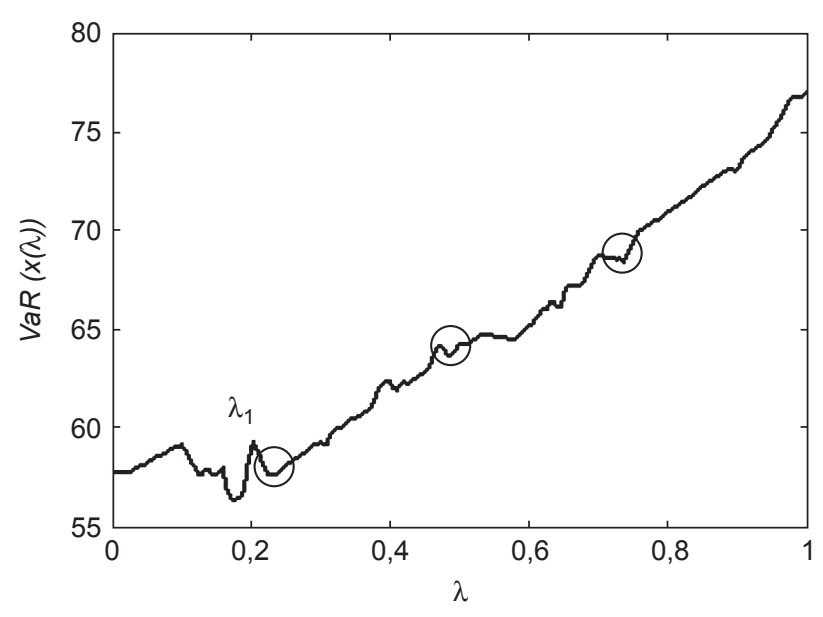

Figura 1. Comportamento do VaR para uma família de carteiras.

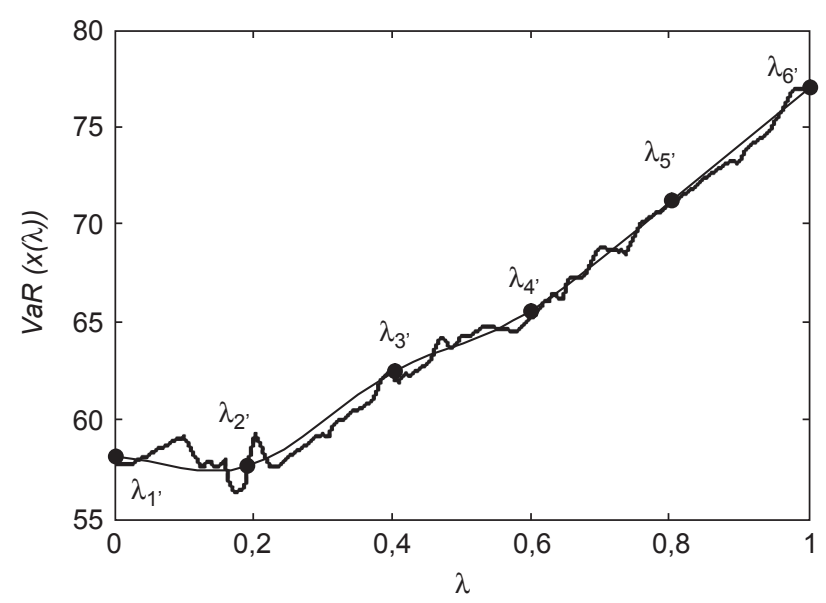

Figura 2. Ajuste de uma função pelo método de aproximação.

locais não é recente. Uma das vantagens na abordagem adotada reside no fato de que as aproximações utilizadas são suficientemente "bem comportadas", possibilitando que algoritmos tradicionais de otimização sejam utilizados para sua minimização. Mas, além disso, o ajuste de curva parte da premissa que os valores da função objetivo obedecem a um modelo estatístico no qual os erros aleatórios não são independentes. Ou seja, se os pontos $\mathrm{x}_{1}$ e $\mathrm{x}_{2}$ estão próximos, os erros de aproximação de $\mathrm{f}\left(\mathrm{x}_{1}\right)$ e $\mathrm{f}\left(\mathrm{x}_{2}\right)$ também estarão próximos. Esta característica é consistente com a estrutura dos problemas de composição de carteiras e, particularmente, com medidas de risco que consideram percentis da distribuição das perdas. As grandes perdas financeiras sofridas por gestores de carteiras devem-se a variações adversas dos retornos e não a pequenas variações na sua composição.
O modelo apresentado a seguir reduz as dificuldades no processo de otimização, provenientes da utilização de estimadores de percentil, apresentando bom ajuste a problemas reais. Embora os cálculos e testes sejam feitos calculando-se o VaR pelo método de série histórica, o modelo proposto é aplicável a qualquer técnica de estimação de percentil.

\section{O método DACE para estimação do VaR}

O pressuposto que norteia este trabalho é que, em um dado instante, é possível determinar estimativas do Valor em Risco para diferentes composições de carteira, o que permite construir uma superfície $S(x)$ que estima a função $\operatorname{VaR}_{\alpha}(x)$. Ainda que sejam conhecidas na literatura diversas técnicas para determinação do VaR de carteiras, a função $\operatorname{VaR}_{\alpha}(x)$ para $x \in \mathfrak{R}^{n}$ não possui uma forma fechada simples a menos de situações elementares.

Rockafellar e Uryasev (2002) apresentaram a seguinte expressão para o cálculo de $\operatorname{VaR}_{\alpha}(x)$. Seja $x \in \mathfrak{R}^{n}$ a composição de uma carteira, e $\mathrm{f}(\mathrm{x}, \mathrm{y})$ a função de perda, em que $y \in \Re^{m}$ é um vetor aleatório de retornos com densidade $\mathrm{p}(\mathrm{y})$. Dado $\alpha \in$ ]0;1[, o $\operatorname{VaR}_{\alpha}(x)$ é definido por

$\operatorname{VaR}_{\alpha}(x)=\min \{\xi \in \mathfrak{R} \mid \varphi(x, \xi) \geq \alpha\}$,

em que

$\varphi(x, \xi)=P(f(x, y) \leq \xi)=\int_{f(x, y) \leq \xi} p(y) d y$

Esta formulação conduz a modelos de otimização lineares de grandes dimensões, porém com o grande inconveniente de admitir conhecida uma distribuição de probabilidades, o que na prática não ocorre.

A técnica aqui apresentada insere-se dentro do que é conhecido em otimização como "métodos de funções aleatórias" (random function methods), em que a função objetivo é tratada como uma realização de um processo estocástico definido a priori, e caracterizado por uma função de correlação entre os valores da função calculados em diferentes pares de pontos (Pardalos et al., 2000), (Jones et al., 1998). A abordagem é bastante utilizada em situações nas quais o custo do cálculo da função objetivo é muito alto, o que não necessariamente ocorre no cálculo das estimativas da função $\operatorname{VaR}_{\alpha}(x)$. Assim, a principal vantagem da metodologia empregada para resolução do problema decorre da interpretação da função objetivo como um processo estocástico com uma estrutura de correlação que reflete o conceito de que carteiras com composições similares devem ter risco semelhante.

Diversos modelos têm sido utilizados para aproximar funções que resultam de cálculos computacionais ou cujo custo de avaliação é alto. As técnicas têm como base uma escolha apropriada de pontos, nos quais a função deve ser calculada, e a seleção de uma superfície que aproxima a função de interesse. Tais técnicas, conhecidas como ajustes DACE (Design and Analysis of Computer Expe- 
riments), têm se mostrado eficientes, tanto no uso para aplicações reais quanto em análises de funções específicas, como a função de Branin, tendo sido desenvolvidas para modelagem de funções não lineares que normalmente ocorrem na engenharia. A técnica teve origem no estudo de problemas oriundos da geologia, e é conhecida como Kriging (Pardalos et al., 2000). São apresentados a seguir os principais conceitos relacionados à técnica. Um estudo mais aprofundado é apresentado por Jones et al. (1998).

Consideram-se observações de uma função $f(),.\left\{\left(w^{(j)} ; y^{(j)}\right)\right\}^{\mathrm{q}}{ }_{\mathrm{j}=1}$, em que $w^{(j)} \in \Re^{n}$ e $y^{(j)}=f\left(w^{(j)}\right)$. O ajuste DACE encontra uma aproximação polinomial $S($.) da função $f($.), que a interpola nos pontos observados, por meio de um modelo do tipo $\sum_{j=1}^{m} \alpha_{j} f^{(j)}(w)+e(w)$, no qual os

erros aleatórios $e(w)$ são normalmente distribuídos com média zero, variância constante, $\sigma^{2}$, e além disso são correlacionados. A correlação entre os erros $\varepsilon\left(w^{(*)}\right)$ e $\varepsilon\left(w^{(j)}\right)$ depende da distância entre os pontos, de tal forma que quanto mais próximo estiver o ponto $w^{(*)}$ do ponto $w^{(j)}$, menor será a diferença entre $\varepsilon\left(w^{(*)}\right)$ e $\varepsilon\left(w^{(j)}\right)$ e maior será a correlação entre eles. De acordo com a distância utilizada é possível construir diferentes estruturas de correlação entre os erros $\varepsilon\left(w^{(j)}\right)$ e $\varepsilon\left(w^{(k)}\right)$ o que permite obter diferentes funções de ajuste à amostra dada. As medidas de correlação usuais são da forma $\sigma^{2} \Sigma$, em que $\Sigma \mathrm{ij}=C\left(w^{(j)}, w^{(k)}\right)=$ $\exp \left[-d\left(w^{(j)}, w^{(k)}\right)\right]$. A medida de distância utilizada é dada por uma função que prioriza, por meio de parâmetros $\theta_{h} \mathrm{e}$ $p_{h}$, as variáveis mais "ativas" na previsão:

$$
d\left(w^{(j)}, w^{(k)}\right)=\sum_{h=1}^{n} \theta_{h}\left|w_{h}^{(i)}-w_{h}^{(k)}\right|^{p_{h}} \quad\left(\theta_{h} \geq 0, p_{h} \in[1,2]\right) .
$$

Embora os parâmetros $\theta_{h}, p_{h}$ e $\sigma^{2}$ possam ser obtidos por meio de técnicas estatísticas de estimação, admite-se, para este estudo, que $p_{h}=2$, numa abordagem similar a Queipo et al. (2002).

Uma vez determinados $\sigma^{2}, \theta_{h}$ e $p_{h}$, o estimador não viesado de mínimos quadrados para o $\mathrm{f}\left(w^{*}\right)$ será dado por (Lophaven et al., 2002):

$$
\hat{f}(w *)=\sum_{j=1}^{m} \beta_{j}^{*} f^{j}(w *)+r^{\prime} \Sigma^{-1}(y-F \beta *)
$$

em que:

$\beta=\left(F^{T} \Sigma^{-1} F\right)^{-1} F^{T} \Sigma^{-1} y$

$r$ é o vetor de correlações entre os erros em $w^{*}$ e os erros nos pontos da amostra. Ou seja, $r_{i}\left(w^{*}\right)=C\left(w^{*}, w^{(i)}\right)$, para uma determinada escolha da distância;

$\mathrm{S}$ é a matriz de correlação entre os erros dos pontos da amostra, ou seja, $\Sigma_{j k}=C\left(w^{(j)}, w^{(k)}\right)$;

$y$ é o vetor dos valores observados da função para a amostra;

F é a matriz com os valores das funções calculados nos pontos da amostra, dada por: $F_{i j}=f^{i j}\left(w^{(j)}\right)$.
Deve-se observar que, a rigor, a função $\operatorname{VaR}_{\alpha}(x)$ não é conhecida, sendo possível determinar apenas estimadores da função. Uma estimativa nada mais é que uma observação de uma variável aleatória, de tal sorte que a aplicação do método DACE para aproximação da função $\operatorname{VaR}_{\alpha}(x)$ é natural. Considera-se uma seqüência de observações $\left\{\left(x^{(j)} ; y^{(j)}\right)\right\}^{q}{ }_{j=1}$, em que $x^{(j)} \in \mathfrak{R}^{n}$ e $y^{(j)}=M_{[\alpha T: T]}\left(-R^{t} x\right)$ a partir da qual é construída uma aproximação $S(x)$.

\section{Seleção da amostra e validação do modelo}

O emprego de um conjunto de observações adequado é essencial para garantir uma boa aproximação da função. No delineamento de experimentos clássicos, utilizados em experimentos físicos, há a preocupação de ocupar o espaço de busca por meio de técnicas como blocagem, replicação e aleatorização. Porém, de acordo com Sacks et al. (1989), no caso de experimentos computacionais, o emprego destas técnicas não acrescenta informação relevante. Diversos autores têm estudado o problema de obtenção de uma amostra apropriada para experimentos computacionais. Simpson et al. (2001) analisam o impacto do uso de diferentes estratégias de amostragem no delineamento e análise de experimentos computacionais. Bates et al. (2003) estudam técnicas computacionalmente eficientes para geração de amostras.

Neste artigo, são avaliados três métodos para geração de amostras: no primeiro, denominado aleatório, os pontos são gerados por meio de uma distribuição uniforme

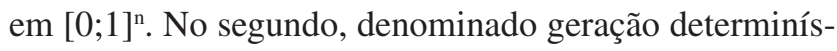
tica, cada face do hipercubo $[0 ; 1]^{\mathrm{n}}$ é subdividida em $\mathrm{P}$ subintervalos que dão origem a hipercubos cujos vértices são escolhidos para amostra. Finalmente, na geração por hipercubo latino (LHS - Latin Hypercube Sampling) as $\mathrm{n}$ componentes de cada vetor são geradas aleatoriamente dentro de estratos pré-determinados. A seguir, as componentes geradas são selecionadas aleatoriamente, de forma a compor um vetor de dimensão n (McKay et al., 1979).

Após ter sido gerada uma amostra, é necessário avaliar sua qualidade; Jones et al. (1998) sugerem o uso de técnicas de cross validation para medir a adequação da superfície construída a partir de uma amostra. Simpson et al. (2001) consideram um conjunto de nval pontos de validação gerados aleatoriamente, para os quais são calculados o erro absoluto máximo (EAM) e o erro quadrático médio (EQM), respectivamente:

$$
E A M=\max _{j=1,2, \ldots, \text { nval }}\left\{\left|y_{i}-\hat{y}_{i}\right|\right\} \quad E Q M=\frac{\sum\left(y_{i}-\hat{y}_{i}\right)^{2}}{\text { nval }}
$$

em que $\hat{y}$ e $y_{i}$ correspondem aos valores obtidos para as superfícies aproximadoras $\mathrm{S}\left(\mathrm{x}^{(\mathrm{i})}\right)$ e para $\operatorname{VaR}_{\alpha}\left(\mathrm{x}^{(\mathrm{i})}\right)$ (segundo a estimativa por série histórica).

A fim de ilustrar graficamente o que foi apresentado, são exibidas as superfícies construídas para os diferentes 
tipos de amostragem. Considerou-se uma carteira hipotética com três ativos financeiros negociados na Bolsa de Mercadorias e de Futuros (BM\&F), a saber, contratos Futuros de Cupom Cambial com maturidade para 5, 35 e 65 dias corridos. Fixou-se a composição dos contratos com maturidade de 65 dias, ou seja, tomou-se $x_{3}=30 \%$ e os valores das demais variáveis foram então gerados por meio das diferentes técnicas de amostragem. Para calcular o Valor em Risco diário, ao nível de confiança de $95 \%$, empregaram-se 994 observações e o estimador de série histórica. As variáveis $\mathrm{x}_{1}$ e $\mathrm{x}_{2}$ representam as frações do capital investido em títulos com maturidade de 5 e 35 dias respectivamente. Na Figura 3, são apresentadas as superfícies aproximadoras obtidas, utilizando-se respectivamente as técnicas de amostragem aleatória, determinística e segundo a geração por hipercubos latinos. Cada uma das amostras utilizadas para a obtenção das superfícies possuía 100 pontos que, na Figura 3, correspondem aos pontos destacados sobre cada superfície. A fim de simplificar a representação gráfica, a restrição $\sum_{j=1}^{n} x_{j}=1$ foi relaxada.

Naturalmente, ao considerar amostras que envolvem a geração de números aleatórios, obtêm-se superfícies mais irregulares do que no caso em que a geração de pontos é determinística. Esta característica parece estar ligada ao fato de que nas amostras com aleatoriedade pode ocorrer a concentração de pontos em particulares regiões, contrariamente ao caso determinístico. Do ponto de vista de otimização, esta irregularidade é indesejável, uma vez que a qualidade das soluções obtidas pelos métodos numéricos depende fundamentalmente da suavidade da função objetivo. Deve-se destacar que a motivação do trabalho foi justamente reduzir a irregularidade da função $\operatorname{VaR}_{\alpha}(x)$.

A qualidade da aproximação obtida também pode ser analisada por meio do erro quadrático médio (EQM). Tomou-se, para os exemplos acima, uma amostra de validação com 100 pontos gerados aleatoriamente e calculou-se o valor do erro para cada caso. A amostragem aleatória e a geração por hipercubos latinos forneceram erros quadráticos médios da ordem de $6,3 \times 10^{-5}$ e $3,9 \times 10^{-5}$, respectivamente, enquanto que, para a amostragem determinística, o erro foi de aproximadamente $1,7 \times 10^{-5}$ (valores aproximados para uma casa decimal).

\section{Resultados computacionais}

O método proposto foi utilizado em um problema real do mercado brasileiro. Considerou-se uma série de taxas de retorno de ações de aproximadamente 3 anos, que forneceu 718 observações diárias. Foram selecionadas dez ações do Índice Bovespa e também o próprio índice, totalizando onze ativos. Calculou-se, a seguir, o Valor em Risco a um nível de $5 \%$ pelo método de séries históricas. Todos os cálculos foram realizados com o aplicativo MATLAB.
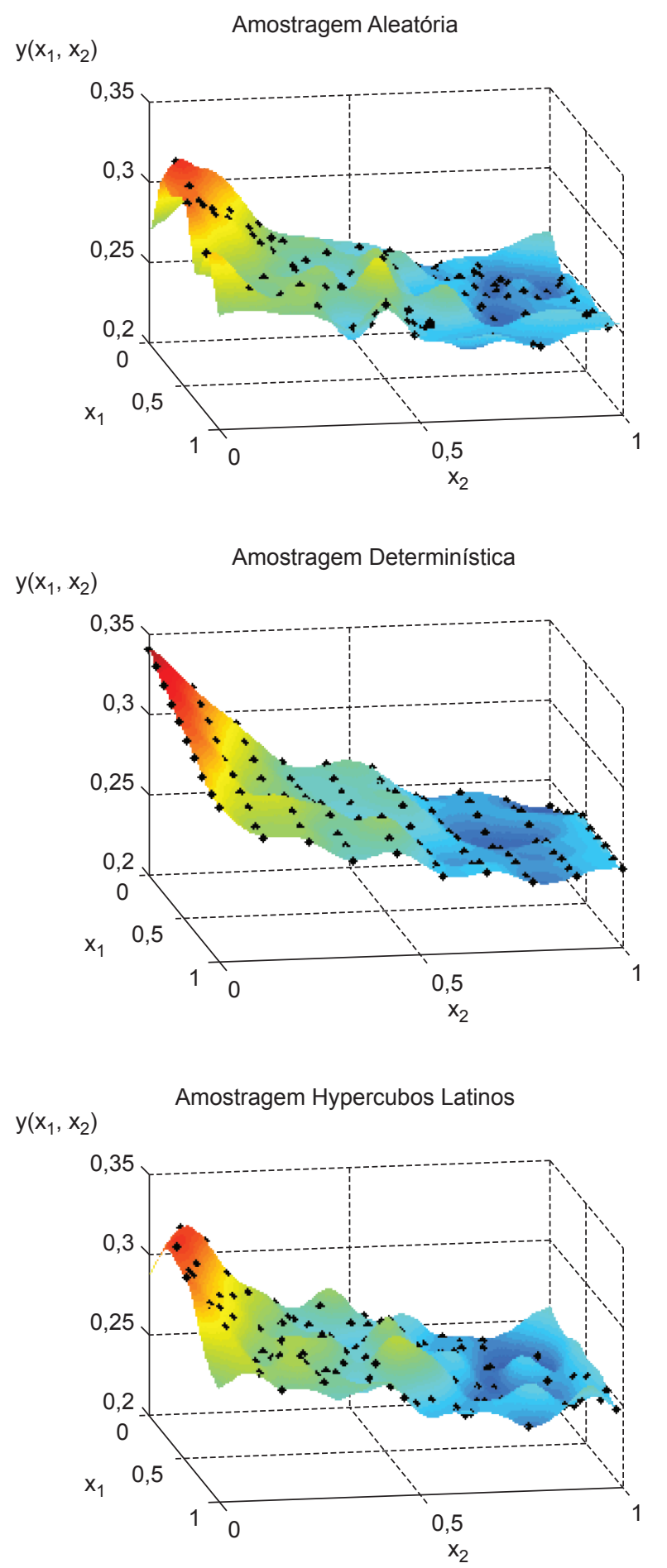

Figura 3. Aproximação gerada pelo método DACE utilizando-se amostragem aleatória, determinística e segundo a geração de hipercubos latinos.

Antes de resolver o problema com os onze ativos, procurou-se realizar um estudo empírico para identificar qual a amostragem mais apropriada ao problema em questão, bem como a quantidade de pontos necessários para realizar uma aproximação aceitável. Assim, verificou-se, 
primeiramente, a relação entre o número de ativos (n), o número de pontos da amostra para gerar a superfície (Namostra) e o tipo de amostra. Os pontos para cálculo dos erros de avaliação foram gerados aleatoriamente, sendo empregados para Namostra pontos, ou seja, a mesma quantidade de pontos utilizada para construir a superfície.

A análise foi realizada para instâncias de tamanho reduzido do problema. Variou-se a dimensão dos vetores considerados (n), bem como o tamanho da amostra para geração de superfície, tendo-se assumido Namostra $=\mathrm{m}^{\mathrm{n}}$. Adotou-se a seguinte denominação para as técnicas de amostragem: Det é a amostragem determinística, $\mathrm{Al}$ a amostragem aleatória e Hip a geração por hipercubos latinos. Os resultados são apresentados na Figura 4.

Observe que os erros diminuem à medida que são utilizados mais pontos para a construção da superfície. É claro que o aumento no número de pontos acarreta um significativo acréscimo do custo computacional da determinação da superfície aproximadora, o que pode tornar o método proibitivo. Assim, a efetiva escolha de um particular número de pontos deve levar em conta a relação entre tempo disponível e qualidade desejável da aproximação. Ainda que não se possa concluir estatisticamente que a amostra determinística é a mais adequada, uma vez que seria necessária a geração de vários testes, para cada par (n, m), os erros de aproximação no caso deste tipo de geração de pontos são claramente inferiores aos erros da geração aleatória para tamanhos de amostra maiores. Por sua vez os resultados obtidos com a geração por hipercubos latinos não apresentam comportamento significativamente superior ao caso da amostragem determinística. Estes fatos, somados ao interesse em obter uma superfície com poucos mínimos locais, levaram à escolha da amostragem determinística para o tratamento do problema de interesse.

Não é objetivo deste trabalho analisar o método em termos de velocidade de processamento, mas sim avaliar

\begin{tabular}{|r|l|r|r|c|}
\hline \multicolumn{5}{|c|}{ EAM $\left(\times 10^{3}\right)$} \\
\hline$n$ & $i$ & \multicolumn{3}{|c|}{$m$} \\
& & \multicolumn{1}{|c|}{3} & \multicolumn{1}{c|}{5} \\
\hline & Det & 1,9 & 1,4 & 1,4 \\
2 & Hip & 5,3 & 13,9 & 3,1 \\
& Al & 9,1 & 119,2 & 3,7 \\
\hline & Det & 34,2 & 2,4 & 1,9 \\
3 & Hip & 9,3 & 31,0 & 3,8 \\
& Al & 26,3 & 12,6 & 3,3 \\
\hline & Det & 33,3 & 5,0 & 3,4 \\
4 & Hip & 26,2 & 10,1 & 4,4 \\
& Al & 93,1 & 5,9 & 5,6 \\
\hline \multirow{3}{*}{5} & Det & 50,4 & 5,4 & 4,0 \\
& Hip & 29,2 & 5,9 & 5,2 \\
& Al & 31,6 & 6,8 & 5,4 \\
\hline
\end{tabular}

\begin{tabular}{|r|l|r|r|c|}
\hline \multicolumn{5}{|c|}{ EQM $\left(\times 10^{5}\right)$} \\
\hline$n$ & $i$ & \multicolumn{3}{|c|}{$m$} \\
& & \multicolumn{1}{c|}{4} \\
\hline & Det & 0,06 & 0,03 & 0,03 \\
2 & Hip & 0,51 & 1,03 & 0,09 \\
& Al & 1,35 & 45,71 & 0,06 \\
\hline & Det & 27,05 & 0,07 & 0,04 \\
3 & Hip & 0,76 & 1,76 & 0,06 \\
& Al & 5,40 & 0,44 & 0,07 \\
\hline & Det & 23,54 & 0,27 & 0,07 \\
4 & Hip & 2,33 & 0,18 & 0,07 \\
& Al & 8,12 & 0,15 & 0,08 \\
\hline & Det & 33,88 & 0,11 & 0,03 \\
5 & Hip & 2,09 & 0,14 & 0,04 \\
& Al & 2,63 & 0,13 & 0,06 \\
\hline
\end{tabular}

Figura 4. Erro absoluto máximo (EAM) e erro quadrático médio (EQM) em função do número de ativos, do tipo e tamanho da amostra. seu comportamento na resolução de problemas reais, razão pela qual procurou-se estudar o comportamento das carteiras de mínimo Valor em Risco, obtidas pelo método proposto. Note que não é possível obter expressões analíticas para descrever as soluções ótimas, pois se parte do pressuposto que a função objetivo é uma realização de um processo estocástico e que a determinação do conjun-

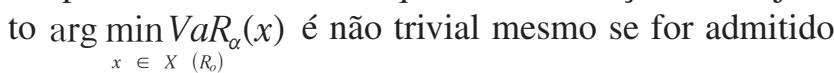

que $\operatorname{VaR}_{\alpha}(x)$ é determinística. Não é viável, conseqüentemente, comparar soluções ótimas com soluções teóricas, ou mesmo, com resoluções resultantes de implementações de algoritmos de otimização que interpretam o Valor em Risco como uma função determinística. A alternativa é, portanto, averiguar como as soluções se comportam quando comparadas com soluções de outro modelo de composição de carteiras.

A abordagem usual, aqui adotada, para a análise do problema, consiste em construir a fronteira de eficiência à luz da específica medida risco empregada, neste caso, o Valor em Risco. A fronteira de eficiência representa a relação risco retorno associada a um investidor que considera o risco como a sua medida de utilidade (a ser minimizada). É natural estabelecer como parâmetro de referência o modelo clássico de Markowitz, que originou o estudo destas fronteiras, e que assume que o risco da carteira é determinado pela variância do retorno. Ao adotar tal medida de risco, o problema resultante é de fácil resolução, reduzindo-se a um problema de programação quadrática. Espera-se que o modelo de otimização de Valor em Risco tenha um comportamento similar ao modelo de Markowitz, em termos de estabilidade da solução ao longo do tempo e, também, quanto à relação risco retorno, expressa em termos da fronteira de eficiência. Naturalmente não há interesse em uma comparação direta entre os dois modelos a fim de identificar qual o melhor, uma vez que se destinam a investidores distintos, que possuem diferentes posturas frente ao risco.

Para gerar a superfície que aproxima o Valor em Risco, foram utilizados 286 pontos, obtidos por meio da amostragem determinística. A fronteira eficiente - que relaciona risco e retorno - foi então construída a partir de diferentes valores de retorno. Exigia-se que o retorno das carteiras fossem iguais a um conjunto de retornos pré estabelecidos, $R=\left\{R_{k}\right\}^{200}{ }_{k=1}$, portanto, foram resolvidos diversos problemas cujos conjuntos de pontos viáveis eram dados por:

$X\left(R_{k}\right)=\left\{x \in \mathfrak{R}^{n} \mid \sum_{j=1}^{n} x_{j}=1 \sum_{j=1}^{n} E\left(r_{j}\right) x_{j}=R_{k} \quad x_{j} \geq 0 \forall j\right\}$.

Os resultados são apresentados graficamente na Figura 5, em que o eixo vertical representa o retorno exigido, $R_{k}$, e o eixo horizontal o Valor em Risco, ambos expressos em percentual. 


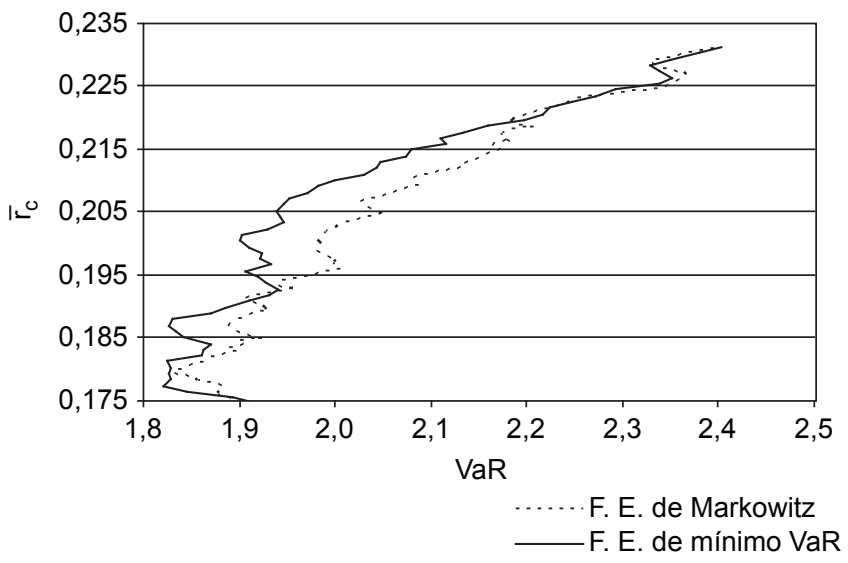

Figura 5. Fronteira do Valor em Risco para modelo de mínimo VaR e Markowitz.

Para cada valor de retorno mínimo exigido, exibe-se o valor em risco das carteiras ótimas obtidas por meio dos dois modelos. Os pontos sobre a curva contínua representam os pares (Retorno médio;Valor em Risco) da carteira ótima de mínimo Valor em Risco, obtida quando se exige pelo menos aquele específico retorno. A curva pontilhada apresenta o Valor em Risco da carteira de mínima variância determinada pelo modelo de Markowitz para o mesmo valor de retorno médio. Assim as curvas representam os pares: $\left(R_{0} ; \operatorname{VaR}\left(x^{*}\left(\mathrm{R}_{0}\right)\right)\right)$ em que $x^{*}\left(R_{0}\right) \in \arg \underset{x \in X\left(R_{0}\right)}{\min \operatorname{Var}}(x)$

e $\left(R_{0} ; \operatorname{VaR}\left(x^{M}\left(\mathrm{R}_{0}\right)\right)\right)$ sendo $x^{M}\left(R_{0}\right)$ a solução do modelo de Markowitz, isto é, $x^{M}\left(R_{0}\right) \in \arg \min _{x \in X} x^{t} \sum_{(R)} x(x)$ em que $\Sigma$ é

a matriz de covariância dos retornos.

Uma característica desejável e importante na gestão de carteiras é a sua estabilidade, ou seja, espera-se que não seja necessário realizar muitas alterações na composição das carteiras ao longo do tempo, devido aos altos custos de reestruturação e necessidade de liquidez. A premissa de inexistência de custos operacionais, presente na maioria dos modelos de composição de carteiras, não reflete a realidade e é usualmente uma das críticas a estes modelos. Para avaliar o desempenho do modelo de mínimo Valor em Risco, sob esta ótica, utilizou-se uma abordagem usual em finanças que considera janelas de tempo. A partir de um histórico de retornos de comprimento fixo (uma janela), gera-se a carteira ótima. A cada período de cálculo, a observação mais antiga é excluída do histórico e acrescenta-se uma nova observação referente ao último retorno observado, repetindo-se o processo de otimização. Do total de 718 retornos disponíveis utilizou-se um histórico fixo de 466 observações e foram feitas 252 determinações da solução ótima, alterando-se o histórico em um dia. As carteiras ótimas foram calculadas, para os dois modelos, fixando-se o retorno mínimo esperado em $0,2 \%$. As figuras que seguem (Figuras 6 e 7), usuais na

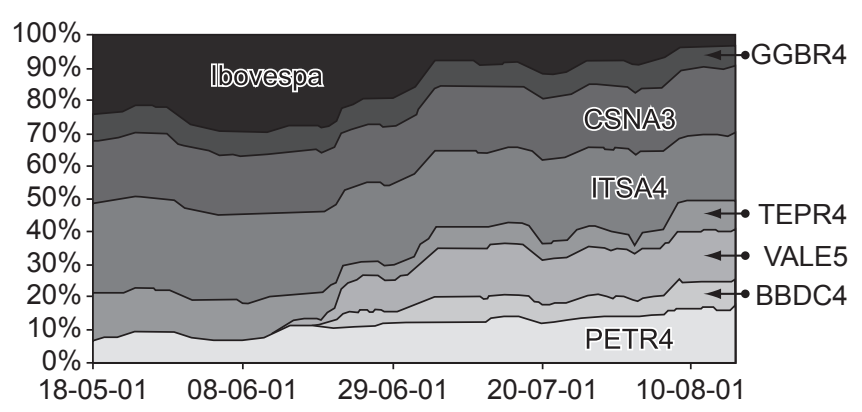

Figura 6. Evolução temporal da composição da carteira ótima segundo o modelo de mínimo VaR.

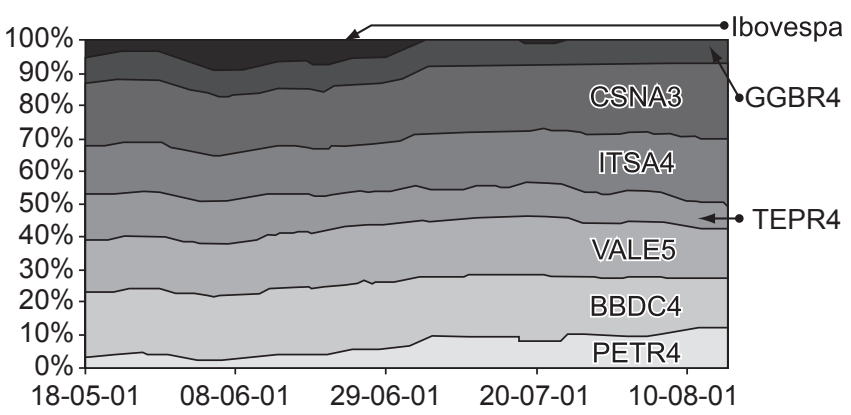

Figura 7. Evolução temporal da composição de carteira ótima segundo o modelo de Markowitz.

literatura de finanças, resumem os resultados obtidos. Estes gráficos descrevem a evolução da composição da carteira ao longo do tempo, quando se toma a decisão com base, exclusivamente, no modelo de otimização. Considera-se que modelo é empregado diariamente, sendo que em cada dia a nova carteira ótima é determinada utilizando-se a série de dados atualizada até o dia precedente, excluindo-se a observação mais antiga. No eixo vertical são apresentados os percentuais de cada ativo que compõem a carteira e cada textura refere-se a um particular ativo. Por exemplo, os dados relativos ao Índice Bovespa, apresentados com a tonalidade mais escura, na parte superior do gráfico, indicam que na data inicial $23 \%$ da carteira era composta por este ativo. À medida que o tempo passa, este valor se altera, até que na data final representa apenas 2,5\%. Estudou-se um período de três meses.

Embora estes resultados não permitam tirar conclusões quanto à superioridade de um modelo ou outro, visto que dependem da liquidez dos mercados, volumes financeiros envolvidos e custos específicos de compra ou venda de ativos, pode-se observar que carteiras obtidas pelo modelo de mínimo Valor em Risco são menos estáveis do que as obtidas pelo modelo de Markowitz. Verifica-se que, para carteiras obtidas segundo o modelo de Markowitz, a maior alteração da composição de um ativo entre dois dias consecutivos foi de $2 \%$, enquanto que, para carteiras 
obtidas segundo o modelo de mínimo VaR, a alteração máxima é de 6\%. Esta questão deve ser levada em consideração quando da opção pela composição de carteiras segundo um modelo ou outro. Deve ser ponderado se a redução do Valor em Risco obtida pelo modelo justifica os custos referentes à menor estabilidade das carteiras, salientando-se que o modelo de Valor em Risco presume que o investidor se preocupa com a perda máxima que pode ocorrer com uma dada probabilidade. Isto significa que um eventual aumento de custos operacionais não necessariamente refletirá uma perda para o investidor, uma vez que mantém sua carteira dentro de níveis aceitáveis de risco, uma das exigências dos comitês de avaliação de riscos nacionais e internacionais.

\section{Conclusões}

Este trabalho estudou o problema de determinação de carteiras de ativos financeiros de mínimo risco, quando se consideram medidas de risco não convexas. A medida selecionada para estudo, o Valor em Risco, embora bastante difundida e utilizada para aferição de risco, não é empregada para determinação de carteiras ótimas, devido às dificuldades de implementação do modelo de otimização subjacente. Para contornar tais inconvenientes, foi proposta a aplicação de uma ferramenta de otimização baseada em aproximação estocástica que possibilitou a determinação de carteiras de mínimo Valor em Risco. Seu uso permitiu que o problema de composição de carteiras de mínimo Valor em Risco fosse resolvido de forma simples, principalmente no que se refere à determinação de mínimos globais.

Foram também apresentados alguns estudos comparativos entre as soluções e as fronteiras eficientes obtidas pelo modelo de Markowitz e pelo modelo de mínimo Valor em Risco. Destes estudos depreende-se que as soluções obtidas pelos dois modelos possuem características distintas, que devem sem contempladas pelos investidores no momento de determinação de suas carteiras. Questões de natureza qualitativa e individual tornam impraticável a determinação do "melhor modelo" a menos de uma seleção ex-ante. Se o que se pretende é determinar a carteira de mínima variância, o modelo apropriado será o de Markowitz. Caso se deseje encontrar a carteira de mínimo Valor em Risco, o modelo adequado será o que minimiza esta medida de risco. Análises multidimensionais que consideram risco e custos operacionais, mais uma vez dependerão de uma postura específica do investidor. Mesmo que se opte por uma particular abordagem, a análise da fronteira eficiente, bem como da evolução temporal da composição das carteiras, poderá contribuir para uma compreensão mais aprofundada das componentes do risco associado àquela carteira. A redução da participação de um ativo na carteira reflete um acréscimo na sua importância relativa no risco global da carteira, que não necessariamente se traduz numa compensação em termos de retorno. Conseqüentemente, ainda que não se determine a priori uma medida de risco, destas análises se poderá inferir, ao menos qualitativamente, relações capazes de direcionar a tomada de decisão.

Destaque-se que a abordagem aqui proposta possui aplicações em diversos problemas de gestão financeira na indústria, que incluem até mesmo a determinação de carteira de produtos. Neste caso, pode-se considerar, por exemplo, que o risco esteja associado ao lucro e que a incerteza decorra exclusivamente de flutuações de preços. Ao pressupor que a receita é função das quantidades produzidas, as variáveis de decisão passam a ser as quantidades, ou percentuais (financeiros) produzidos de cada produto. O risco pode, então, ser definido como um percentil associado à distribuição de probabilidades do lucro, isto é, o Valor em Risco. Torna-se assim natural obter a carteira de produtos que minimiza o Valor em Risco do lucro.

Entre os trabalhos futuros a serem realizados estão estudos sobre o desempenho do método na composição de carteiras que utilizam outras medidas de risco. É natural também averiguar o impacto da escolha de particulares estruturas de correlação na definição do processo estocástico que determina a superfície que aproxima o Valor em Risco, assim como analisar diferentes aproximações estocásticas.

\section{Referências Bibliográficas}

ACERBI, C.; TASCHE, D. On the coherence of expected shortfall. Journal of Banking and Finance, v. 26, n. 7, p. 1487-1503, 2002.

ARTZNER, P., et al. Coherent measures of risk. Mathematical Finance, v. 9, p. 203-228, 1999.

BATES, S. J.; SIENZ, J.; LANGLEY, D. S. Formulations of the Audze-Eglais Uniform Latin Hypercube design of experiments. Advances in Engineering Software, v. 34, n. 8, p. 493-506, 2003.
BERTSIMAS, D.; LAUPRETE, G. J; SAMAROV, A. Shortfall as a risk measure: properties, optimization and applications. Journal of Economic Dynamics \& Control, v. 28, n. 7, p. 1353-1381, 2004.

EMBRECHTS, P. Extreme Value Theory: potential and limitations as an integrated risk management tool. Derivatives Use, Trading and Regulation, v. 6, p. 449-456, 2000.

JONES, D. R.; SCHONLAU, M.; WELCH, W. J. Efficient global optimization of expensive black-box functions. 
Journal of Global Optimization, v. 13, n. 4, p. 455-492, 1998.

JORION, P. Value at Risk: the new benchmark for controlling derivatives risk. New York: McGraw Hill, 1997.

JÚDICE, J. J., RIBEIRO, C. O., SANTOS, P. J. Análise Comparativa dos Modelos de Selecção de Carteiras de Ações de Markowitz e Konno. Investigação Operacional, v. 23, n. 2, p. 211-224, 2003.

KIBZUN, A.; KIPP, R. Discrete approximation in quantile problem of portfolio selection, In: URYASEV, S.; PARDALOS, P. M. (Ed.): Stochastic optimization algorithms and applications. Boston: Kluwer, 2001. p. 121-135.

KONNO, H.; YAMAZAKI, H. Mean-absolute deviation portfolio optimization model and its application to Tokyo stock market. Management Science, v. 37, n. 5, p. 519-531, 1991.

LOPHAVEN, S. N.; NIELSEN, H. B.; SØNDERGAARD, J. DACE: a matlab kriging toolbox. Denmark: Informatics and Mathematical Modelling, Technical University of Denmark, 2002. 28 p. (Technical Report, IMM-TR2002-12).

LUENBERGER, D. G. Investment Science. New York: Oxford University Press, 1998.

MARKOWITZ, H. M. Portfolio Selection. Journal of Finance, v. 7, n. 1, p. 77-91, 1952.

MCKAY, M. D.; CONOVER, W. J.; BECKMAN, R. J. A comparison of three methods for selecting values of input variables in the analysis of output from a computer code. Technometrics, v. 21, n. 2, p. 239-245, 1979.

MCNEIL, A. J. Calculating quantile risk measures for financial time series using extreme value theory. 1998. Disponível em: <http://e-collection.ethbib.ethz.ch/show? type $=$ bericht $\& n r=85>$. Acesso em: 2 de agosto de 2005 .
PARDALOS, P. M.; ROMEIJN, H.; TUY, H. Recent developments and trends in global optimization. Journal of Computational and Applied Mathematics, v. 124, n. 1-2, p. 209-228, 2000.

PFLUG, G.; GAIVORONSKI, A. A. Value-at-risk in portfolio optimization: properties and computational approach. Journal of Risk, v. 7, n. 2, p. 1-31, 2005.

QUEIPO, N.V., et al. Surrogate modeling-based optimization for the integration of static and dynamic data into a reservoir description. Journal of Petroleum Science and Engineering, v. 35, n. 3-4, p. 167-181, 2002.

RIBEIRO, C. O.; GUEDES, M. C. M.; SANTOS, J. Uma análise comparativa de modelos de gestão de carteiras de investimento baseadas no modelo Cvar (Var Condicionado). In: CONGRESSO ANUAL DA SOCIEDADE PORTUGUESA DE ESTATÍSTICA, 9, 2001, Ponta Delgada. Novos Rumos em Estatística, Ponta Delgada, Sociedade Portuguesa de Estatística, 2002. p.327-340.

ROCKAFELLAR, R. T.; URYASEV, S. Optimization of conditional value-at-risk. Journal of Risk, v. 2, n. 3, p. 21-41, 2000.

Conditional value-at-risk for general loss distributions. Journal of Banking and Finance, v. 26, n. 7, p. 1443-1471, 2002.

SACKS, J., et al. Design and analysis of computer experiments. Statistical Science, v. 4, n. 4, p. 409-435, 1989.

SIMPSON, T. W., LIN, D. K. J., CHEN, W. Sampling strategies for computer experiments, design and analysis. International Journal of Reliability and Applications, v. 2, n. 3, p. 31-85, 2001.

STEINBACK, M. C. Markowitz revisited: mean variance models in financial portfolio analysis, SIAM Review, v. 43, p. 31-85, 2001.

SZEGÖ, G. Measures of Risk. Journal of Banking and Finance, v. 26, n. 7, p. 1253-1272, 2002.

\title{
A CONTRIBUTION TO THE MINIMUM VALUE-AT-RISK PORTFOLIO PROBLEM
}

\begin{abstract}
A model based on stochastic approximation is proposed to minimize the risk of portfolios of financial assets. Value-at-Risk, a widely used risk measure in financial management practices, has rarely been used in optimal portfolio context selection due to difficulties in computational implementation. The proposed model allows the minimum Valueat-Risk portfolio problem to be solved through an approximation of the objective function. This paper analyzes the performance of the model in a portfolio of Brazilian market assets.
\end{abstract}

Keywords: portfolio management, finance, optimization. 